\title{
Double Opposing-Rhomboid Flaps for Closure of a Circular Facial Defect in a Special Position
}

\author{
Hui-Ling Wu $\cdot$ Shu-Jun Le $\cdot$ Shu-Sen Zheng
}

Received: 24 February 2008/Accepted: 4 August 2008/Published online: 17 September 2008

(C) The Author(s) 2008. This article is published with open access at Springerlink.com

\begin{abstract}
Various techniques are used to repair circular defects in special positions on the face. Simple closure will yield unsatisfactory results because of skin tension. As an ideal method of reconstruction, various flaps are used to provide a versatile and safe alternative. The authors applied double opposing-rhomboid flaps, which provided for closure of a circular skin defect without alteration to the original shapes of the vital structures and resulted in an imperceptible scar. With careful designing, 21 patients with circular defects caused by skin tumor excisions to special facial positions have attained effective functional and aesthetic results using this method since 2004.
\end{abstract}

Keywords Circular defect .

Double opposing-rhomboid flaps · Plastic surgery

In plastic surgery, the most common facial defect encountered is a circular skin defect. These defects often lie close to vital structures such as the eyes, nose, ear, or mouth. Without predictably healthy skin, simple closure yields unsatisfactory results. Local excision and reconstruction must be designed carefully to achieve a satisfactory functional and aesthetic result.

H.-L. Wu · S.-J. Le

Department of Plastic Surgery, The First Affiliated Hospital, College of Medicine, Zhejiang University, \# 79 Qingchun Road, Hangzhou 310003, Zhejiang Province, P.R. China

\section{S.-S. Zheng $(\varangle)$}

Department of Hepato-Biliary Surgery, The First Affiliated Hospital, College of Medicine, Zhejiang University, \# 79 Qingchun Road, Hangzhou 310003,

Zhejiang Province, P.R. China

e-mail: shusenzheng@zju.edu.cn
We applied double opposing-rhomboid flaps to reconstruct the skin tumor defects of 21 patients and obtained satisfactory results. Three cases are reported.

\section{Patients and Methods}

This study enrolled 21 patients with circular tumors on their faces. The most common position of the tumor was near the eye, followed by tumors of the nose, lip, ear, and forehead. The pathologic types included seven primary melanomas, two basal cell carcinomas, nine nevuses, and three keratosises. The defects ranged in diameter from 0.7 to $4 \mathrm{~cm}$ (mean, $1.8 \mathrm{~cm}$ ), and in depth from 0.17 to $8.13 \mathrm{~mm}$ (mean, $3.42 \mathrm{~mm}$ ).

The mean patient age was 47 years (range, $17-83$ years). Most of the patients were in generally good condition. Five patients had a diagnosis of hypertension, and six patients were addicted to smoking at the time of surgery.

The flaps were designed after the circular defect according to biopsy of the tumors or after the mass was drawn as a circle with sufficient free margins. The designing of the flaps is shown in Figs. 1-3. First, the flaps were created in the two regions of healthy skin on the opposite side of the circle. Second, the two opposing-rhomboid flaps were orientated according to the degree of surrounding tissue movement. Third, the flap was drawn to make the angle of the rhomboid almost a right-angle and the length of the border almost equal to the radius of the circle.

\section{Results}

By applying double opposing-rhomboid flaps, none of our patients experienced postoperative complications such as necrosis, infection, or deformity of vital structures. All the 
Fig. 1 Reconstruction of the nasal columella using double opposing-rhomboid flaps. (a) Melanoma in the nasal columella before operation. (b) Design of the double opposingrhomboid flaps. (c and d) Reconstructed columella with acceptable shape and imperceptible scar 3 months after operation

Fig. 2 Reconstruction of periorbital region tumor using double opposing-rhomboid flaps. (a) Design of double opposing-rhomboid flaps. (b) View 1 month after operation showing natural features without asymmetry of the eyelid and the head of the eyebrow


Fig. 3 Periorbital region correction using double opposing-rhomboid flaps. (a) Design of double opposingrhomboid flaps. (b) View 1 week after operation showing an excellent result without displacement of the free margin of the eyelid or the canthus the resulting zigzag scars became imperceptible 2 to 6 months after the operation. The postoperative follow-up period ranged from 3 months to 4 years, and none of the patients had local recurrences. 


\section{Case Reports}

\section{Case 1}

A 25-year-old man presented with melanoma that occupied almost all of his nasal columella, resulting in a skin defect about $7 \mathrm{~mm}$ in diameter. Realizing that only upper and lower healthy skin can be used for reconstruction, we designed double opposing-rhomboid flaps as shown in Fig. 1. This patient had acceptable columella shape, and the resulting zig-zag scar became imperceptible 3 months after the operation.

\section{Case 2}

A 45-year-old man presented with a nevus in his right periorbital region measuring about $15 \times 12 \times 8 \mathrm{~mm}$. One rhomboid flap was designed for the bridge of the nose, with the tip located immediately in the middle of the back. This patient also had very natural features, and an excellent result was obtained without asymmetry of the eyelid, eyebrow, or canthus, as observed 1 month after the operation (Fig. 2).

\section{Case 3}

A 62-year-old man presented with a basal cell carcinoma in his right periorbital region measuring about $13 \mathrm{~mm}$ in diameter. His lesion was excised with $5 \mathrm{~mm}$ of free margin. Double opposing-rhomboid flaps were designed to orient opposing parallel straight lines in the relaxed skin tension lines. The flaps fit into the circular defect very well without removal of healthy tissue. This man had very natural features, and an excellent result was obtained without displacement of the free margin of the eyelid and canthus (Fig. 3).

\section{Discussion}

The more common lesions of concern to plastic surgeons include tumors and scars. The incidences of nevus, keratose, and melanoma have increased over the past several decades, and reconstructive techniques have become more critical. One great challenge of excision in the face is its proximity to vital structures such as the eyes, nose, ears, and mouth. The need for wide local excision and reconstruction must be balanced carefully with both functional and aesthetic considerations [1].

A circular defect is one of the most commonly encountered defect types after facial tumor excision, especially after popularization of the Mohs surgical excision [2]. To close a circular skin defect, many flaps have been defined in the literature [3-5]. With these methods, the circular defects often are changed into rhomboid or square shapes by making additional excisions from healthy skin. Alternatively, the circular defects are reserved in original form, and the flaps are changed into the shape of the lesion, again by making an additional excision.

Roggendorf [6] described covering circular defects by three oblong Schwenklappen as a favorable combination of techniques. The negative aspects of other techniques, such as tension, bunching, or reduced blood supply, can be minimized by using different flap shapes. This affords a more equal distribution of the tensions at the donor sites by extending them farther into the surrounding tissues. However, in the special parts of the face, vital structures are always nearby, and the remaining healthy tissue is precious. It is difficult to reconstruct the defect considering the shapes of the vital structures.

Rhomboid flaps are well known and used frequently [710], always for covering defects created by simple excisions in the shape of a rhombus. The closure requires construction of a flap equal in size to the defect due to borrowing of the required tissue from a single adjacent flap donor region limited by the potential for tension and muscle contracture. For a larger defect or defect in a special part of the face $[11,12]$, this is not easy to accomplish.

The rhomboid-to-W principle eliminates many of these problems because the rhomboid defect is converted into a $\mathrm{W}$-shaped scar that is easier to conceal in the natural crease lines. The $\mathrm{W}$-shaped scar has only two sides to each flap and thus less tendency for trap-door scarring. Tissue is borrowed from all four directions, resulting in minimal distortion.

However, in planning the rhomboid-to-W flap, the axis of the $\mathrm{W}$ should follow the crease lines. The availability of skin is assessed in all directions, and the flap is planned considering the amount of tissue to be borrowed in each direction [13]. For the circular defect in special positions on the face, tissue in each direction cannot be used. There is not much tissue to be used with vital structures nearby.

For this reason, the technique of double opposingrhomboid flaps is used to repair the excisional defects. This technique borrows the required tissue from two nonadjacent opposite sides of the defect. The "sharing" of tissue from two opposite regions minimizes tension in that direction and prevents the distortion of anatomic landmarks located along that defect [14]. Furthermore, the angle of the rhomboid is almost a right angle, and the length of the border is almost equal to the radius of the defect. Therefore, the opposing-rhombic flaps fit into the circular defect mostly without extra skin excision.

In addition, four segments of the final suture lines are parallel to the relaxed skin tension lines, which makes 
closure easy, and the resultant scar tissue is easily hidden in the natural creases. Thus excessive tension in repairing donor regions is avoided. The orientation of the final scar and the direction of tissue tension can be controlled by designing the opposing-rhombic flap. This technique may be used more safely for the defect adjacent to vital structures, avoiding displacement of vital structures such as eyelids, eyebrows, nasal alae, and lips.

Case 1 required a nasal columella reconstruction that can be quite challenging. Curing the lesion obviously is the primary goal, but a good cosmetic result also is important. Although local nasal flaps can provide excellent results [1], in this case, no columella tissue remained to be used except the adjacent tissue above and below the lesion. Using this method, the nasal columella was reconstructed with identical height but with no change to the position of the nose tine (Fig. 1).

Melanoma and basal cell carcinoma of the orbit often are located on the upper or lower lids. The skin is thin and overlies the orbicularis oculi muscle and conjunctiva. Maintenance of function is of utmost importance for eye protection [1]. The rhomboid flap frequently is used to reconstruct the defect. It offers ample versatility in flap design and minimal normal tissue loss. By the use of double opposing-rhomboid flaps for reconstruction of the periocular area to orientate opposing parallel straight lines in the relaxed skin tension lines, the patient attained very natural features without the displacement of the free margin of the eyelid, eyebrow, or especially the canthus (Figs. 2 and 3).

The postoperative scars may minimize the contracture because tension is redistributed along the lines of the zigzag closure. To relax skin tension, four of seven segments of the final suture lines are parallel, which makes closure easy, resulting in scar tissue easily hidden in the natural creases.

This technique requires somewhat complicated preoperative planning and drawing. Although two possible sites can be used to prepare the double opposing flaps for placement parallel to the relaxed skin tension lines, the one that will not interfere with or distort adjacent anatomic landmarks should be selected. Also, the degree to which the tissues of the flaps will rotate or glide must be estimated. In some cases, after transposition of the flaps, a minimal dog-ear formation has been observed at one end of the flap, but it was too small to require excision and was resolved by time.

\section{Conclusion}

For reconstruction of small-to-medium-sized distal circular defects at special positions on the face, double opposingrhomboid flap closure provides a versatile and safe alternative when simple closure would yield unsatisfactory results. This method provides closure of circular skin defects without alteration of their original shapes and negates excision of additional healthy skin. It also does not affect functional or aesthetic aspects of vital facial structures. Furthermore, the shape of the final suture lines leave a cosmetically acceptable scar.

Open Access This article is distributed under the terms of the Creative Commons Attribution Noncommercial License which permits any noncommercial use, distribution, and reproduction in any medium, provided the original author(s) and source are credited.

\section{References}

1. Bogle M, Kelly P, Shenaq J, Friedman J, Evans GR (2001) The role of soft tissue reconstruction after melanoma resection in the head and neck. Head Neck 23:8

2. Dzubow LM, Zack L (1990) The principle of cosmetic junctions as applied to reconstruction of defects following Mohs surgery. J Dermatol Surg Oncol 16:353

3. Skaria AM (2006) Rotation flap reconstruction for nasal tip defect after Mohs surgery. Dermatology 213:118

4. Dzubow LM (1988) Mohs surgery report: Design of an appropriate rhombic flap for a circular defect created by Mohs microscopically controlled surgery. J Dermatol Surg Oncol $14: 124$

5. McGeorge BC (1998) Modified rhombic flap for closure of circular or irregular defects. J Cutan Med Surg 3:74

6. Roggendorf E (1980) The oblong parallelogram-shaped "Schwenklappen"-plasty. Plast Reconstr Surg 65:635

7. Teske SA, Kersten RC, Devoto MH, Kulwin DR (1998) The modified rhomboid transposition flap in periocular reconstruction. Ophthal Plast Reconstr Surg 14:360

8. Yanai A, Ueda K, Takato T (1986) Flexible rhombic flap. Plast Reconstr Surg 78:228

9. Lawrence WT (1992) The nasolabial rhomboid flap. Ann Plast Surg 29:269

10. Holt PJ, Motley RJ (1991) A modified rhombic transposition flap and its application in dermatology. J Dermatol Surg Oncol 17:287

11. Bullock JD, Hamdi B (1980) Double rhomboid flap in ophthalmic plastic surgery. Ophthalmic Surg 11:431

12. Gahhos FN, Cuono CB (1990) Double-Z rhombic technique for reconstruction of facial wounds. Plast Reconstr Surg 85:869

13. Becker H (1983) The rhomboid-to-W flap. Ann Plast Surg 11:125

14. Keser A, Sensöz O, Mengi AS (1998) Double opposing semicircular flap: a modification of opposing Z-plasty for closing circular defects. Plast Reconstr Surg 102:1001 